\title{
STABILITY OF TRIMETREXATE, A NEW NON-CLASSICAL ANTIFOLATE, IN INFUSION SOLUTIONS
}

\section{P. L. STETSON*}

Upjohn Center for Clinical Pharmacology and Department of Pharmacology, University of Michigan Medical School, Ann Arbor, MI 48109 (U.S.A.)

\section{U. A. SHUKLA}

Upjohn Center for Clinical Pharmacology and School of Pharmacy, University of Michigan Medical School, Ann Arbor, MI 48109 (U.S.A.)

and

W. D. ENSMINGER

Upjohn Center for Clinical Pharmacology, Department of Pharmacology and Department of Internal Medicine, University of Michigan Medical School, Ann Arbor, MI 48109 (U.S.A.)

(Received October 10th, 1988)

\section{SUMMARY}

A prerequisite for the prolonged infusion of a drug via a totally implanted drug delivery system is that the drug solution must be sufficiently stable at physiological temperatures to endure the time intervals between drug replacement or pump refills. Consequently, the stability of the chemotherapeutic agents can influence the dosing accuracy and ultimately the achievement of the desired therapeutic goal. The chemical stability of the pharmaceutical preparation Trimetrexate (TMQ) glucuronate, a non-classical, lipophilic antifolate, has been characterized. Incubation of TMQ (prepared in sterile water to a concentration of $5.0 \mathrm{mg} / \mathrm{ml}$ ) in sterile, amber glass vials at $37^{\circ} \mathrm{C}$ for 56 days resulted in a degradation rate constant of $0.0134 \pm 0.002$ day $^{-1}$ and a half-life of $51.6 \pm 0.8$ days. The major degradation product has been identified as (2,4-diamino-5-methyl-6-carboxaldehyde)quinazoline. Ten percent TMQ degradation would occur by 7.9 days of incubation under these conditions.

\section{INTRODUCTION}

Trimetrexate, 2,4-diamino-5-methyl-6[(3,4,5-trimethoxyanilino)-methyl]quinazoline (TMQ), was originally synthesized by Elslager and Davoll ${ }^{1}$. It is a potent inhibitor of dihydrofolate reductase (DHFR) [5,6,7,8-tetrahydrofolate:NADP ${ }^{+}$oxido-reductase (EC 1.5.1.3) $]^{2}$, and has demonstrated significant antitumor activity against murine and human cell lines both in vitro and against several murine transplanted tumors ${ }^{3,4}$. TMQ is more lipophilic than the classical antifolate methotrexate (MTX) and has demonstrated an increased uptake into neoplastic cells compared to MTX $^{5}$. Also, TMQ's enhanced lipophilicity may afford its greater penetration into 
the central nervous system (CNS) and, therefore, greater efficacy than MTX against CNS neoplasms.

The importance of antifolate concentration and exposure time in determining toxic and therapeutic effects both in vitro and in vivo in experimental animal tumors has been established ${ }^{6,9}$. Studies in experimental animal systems have also shown that biological response to antifolates is determined by exposure time and is selective for individual tissues ${ }^{10-12}$. These findings suggest a therapeutic rationale for the prolonged infusion of TMQ at rigidly controlled dose rates which achieve steady-state TMQ plasma levels toxic to neoplastic cells but below the toxic threshold of bone marrow cells from that individual patient. With the advent of totally implanted drug delivery systems, therapy involving prolonged intravenous or, in the case of regional chemotherapy ${ }^{13}$, intra-arterial infusions can be accomplished on an out-patient basis with minimal patient risk and significant savings in hospital an patient care expenses.

Portable drug delivery systems, both external and implanted, allow constant drug infusion rates at the desired sites for extended durations. However, a prerequisite for prolonged drug infusion via a totally implanted drug delivery system is that the drug solution must be sufficiently stable at physiological temperatures to endure the time intervals between drug replacement or pump refills. Consequently, the stability of the chemotherapeutic agents can influence the dosing accuracy and ultimately the achievement of the desired therapeutic goal. The present study was designed to characterize the stability of the TMQ drug preparation (Trimetrexate glucuronate) in sterile, amberized glass vials during prolonged incubation ( 8 weeks) at $37^{\circ} \mathrm{C}$.

\section{EXPERIMENTAL}

\section{Materials}

Trimetrexate glucuronate (50 mg TMQ/vial) and 2,4-diamino-5-methyl-6-carboxaldehyde-quinazoline (AMCQ) were obtained from Warner-Lambert/Parke-Davis, Pharmaceutical Research Devision, Ann Arbor, MI, U.S.A. All other chemicals were high-performance liquid chromatography (HPLC) or analytical grade. Amberized glass vials ( $10 \mathrm{ml}$ capacity with rubber septa and crimp closures) and N-methylN-trimethylsilyltrifluoroacetamide (MSTFA) were obtained from Pierce (Rockford, IL, U.S.A.). Sterile plastic (1.0 ml) syringes (Becton, Dickinson \& Co., Rutherford, NJ, U.S.A.) were used for sampling.

\section{Kinetic procedures}

Trimetrexate glucuronate injection $(50-\mathrm{mg}$ vial) was reconstituted in $10.0 \mathrm{ml}$ sterile water to yield a concentration of $5.0 \mathrm{mg} \mathrm{TMQ} / \mathrm{ml}$. The reconstituted solution was aseptically transferred to sealed, sterile, amber glass vials. Triplicate sample solutions were prepared. A $0.2-\mathrm{ml}$ aliquot of the samples was removed from each amber vial and signified the samples at zero time. The vials were then incubated in a constant-temperature water bath at $37^{\circ} \mathrm{C}$ and $0.2-\mathrm{ml}$ aliquots were removed from each vial at the following times: $3,10,14,17,21,25,28,31,39,42,47,49$ and 56 days. All samples were frozen immediately upon acquisition at $-30^{\circ} \mathrm{C}$ in tightly capped plastic tubes until TMQ analysis. 


\section{HPLC assay of TMQ}

TMQ standards, quality control and experimental (incubate) samples were diluted 1:5 with methanol-water $(50: 50, v / v)$ and $2.0 \mu \mathrm{l}$ of the diluted samples were injected onto an HPLC reversed-phase column (Hewlett-Packard MOS $\mathrm{C}_{8}, 10 \mathrm{~cm} \times$ $2 \mathrm{~mm}$ I.D., $5 \mu \mathrm{m}$ ) using the Hewlett-Packard Model 1090 high-performance liquid chromatograph and autosampler. The chromatographic conditions were: mobilephase, methanol-acetonitrile- $0.05 M\left(\mathrm{NH}_{4}\right) \mathrm{H}_{2} \mathrm{PO}_{4}$ buffer $(12: 18: 70, \mathrm{v} / \mathrm{v} / \mathrm{v})$; flowrate, $0.5 \mathrm{ml} / \mathrm{min}$; detector, Hewlett-Packard Model 1040 diode array UV-VIS detector monitoring at $254 \mathrm{~nm}$ wavelength and scanning between 200 and $400 \mathrm{~nm}$. The chromatographic peak area integration was acquired using the Hewlett-Packard 3392A integrator/printer.

Calibration curves were constructed by plotting the integrated peak area of $T M Q$ as a function of the drug concentration. The TMQ concentrations of unknown samples were calculated using the results of a least squares linear regression analysis of the calibration data.

\section{Gas chromatography-mass spectrometry}

The Hewlett-Packard Model 5987A gas chromatography-mass spectrometry (GC-MS) apparatus equipped with both electron impact (EI) and methane chemical ionization (CI) modes of operation was used in the identification of the major product of TMQ degradation. Separation of the trimethylsilyl (TMS) derivatives of TMQ and its degradation products was accomplished using a fused-silica capillary column $(5 \mathrm{~m}$ $\times 0.32 \mathrm{~mm}$ I.D. $)$ coated with a cross-linked methyl silicone liquid phase $(0.17 \mathrm{~mm}$ film thickness). Chromatographic conditions were: injection port and GC-MS interface oven temperatures were $300^{\circ} \mathrm{C}$; GC column oven temperature was programmed to hold at the initial temperature of $80^{\circ} \mathrm{C}$ for $0.1 \mathrm{~min}$, and then increase at a rate of $20^{\circ} \mathrm{C} / \mathrm{min}$ up to the final temperature of $300^{\circ} \mathrm{C}$; for EI operation, the ion source temperature was $250^{\circ} \mathrm{C}$, electron energy was $70 \mathrm{eV}$, and the scan range was from 70 to 600 a.m.u.; for methane $\mathrm{CI}$ operation, the ion source temperature was $200^{\circ} \mathrm{C}$, electron energy was $230 \mathrm{eV}$, and the scan range was from 130 to 600 a.m.u.

Trimethylsilylation of TMQ and its degradation products was accomplished using MSTFA. Aliquots of $0.1 \mathrm{ml}$ of both the zero-time and 56-day incubation samples were vacuum dried and the residue derivatized in $0.25 \mathrm{ml}$ MSTFA for $2 \mathrm{~h}$ at $85^{\circ} \mathrm{C}$. $\Lambda 0.5-\mu \mathrm{l}$ volume of the reaction mixture was injected for GC-MS analysis.

\section{RESULTS AND DISCUSSION}

\section{$T M Q$ assay validation}

Under the described chromatographic conditions, the retention time of TMQ was $2.03 \mathrm{~min}$. The integrated peak areas were directly proportional to the drug concentrations over the range of TMQ concentrations tested $(0.50-5.00 \mathrm{mg} / \mathrm{ml})$.

Triplicate sets of the calibration standards were assayed on each of three consecutive days. Linear regression of the data from each day was obtained. The reproducibility of the daily standard curves had coefficients of variation which ranged between 1.7 and $4.8 \%$.

The accuracy and precision of the method were assessed by seeding quality control samples at drug concentrations of 1.00 and $4.00 \mathrm{mg} \mathrm{TMQ} / \mathrm{ml}$. Triplicate 


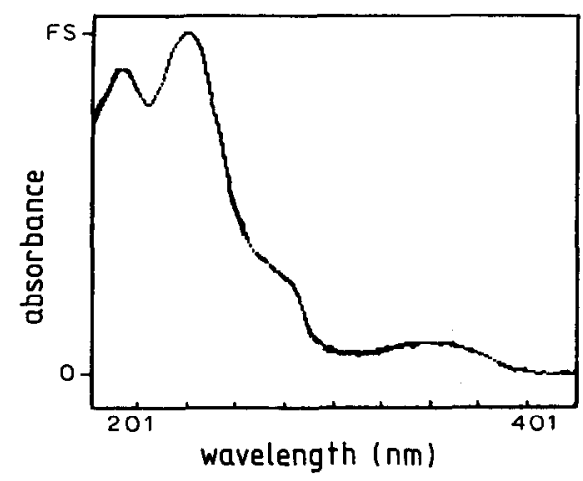

Fig. 1. Results of peak purity evaluation of TMQ peak in HPLC assay procedure. Peak purity is confirmed by three perfectly superimposed UV-VIS absorption spectra.

quality control samples were assayed on each of three consecutive days. The precision of the assay was found to have coefficients of variation ranging between 1.9 and $4.4 \%$. The concentration means for the seeded control samples were found to be within $-1.3-+3.2 \%$ of the theoretical values.

\section{$T M Q$ assay specificity}

The purity of the TMQ peak in the HPLC assay described here was confirmed with a peak purity evaluation program available on the HP 1090 HPLC system equipped with the HP 1040 diode array detector. This program automatically collects UV-VIS absorption spectral data at the upslope, apex and downslope of an eluting peak. In the peak purity analysis, the upslope spectrum of a peak is recorded and becomes a reference spectrum. The apex spectrum of the peak then becomes the reference and the upslope spectrum is discarded. The downslope spectrum of the peak is then plotted against the reference (apex) spectrum. Finally, the end-of-peak spectrum is plotted against the reference (apex) spectrum. Peak purity is established by three perfectly superimposed spectra.

When applied to the TMQ incubation samples, three superimposed spectra

$t=$ zero time $\quad t=21$ days $\quad t=56$ doys

B)
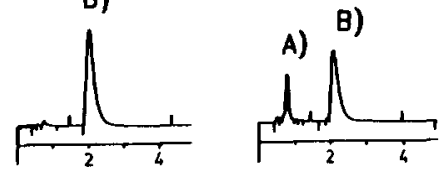

A) B)

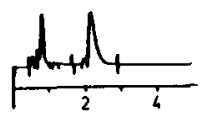

RETENTION TIME (MINUTES)

Fig. 2. HPLC chromatograms (2- $\mu$ l injections) of zero-time, 21-day and 56-day incubation samples showing TMQ (peak B) disappearance and degradation product (peak A) accumulation with increasing incubation time. 
(Fig. 1) of the drug peak (peak B in Fig. 2) resulted, thus verifying that the TMQ peak was uncontaminated by any potentially coeluting products.

\section{Degradation kinetics}

The integrated peak areas of the incubated samples were compared to those resulting from injections of standard solutions. The concentration versus time (in days) data were then entered on semi-log plots as shown in Fig. 3. The stability profile of TMQ can be described by the simple monoexponential decay as given in eqn. 1:

$$
C_{t}=C_{0} \exp (-k t)
$$

where $C_{0}$ is the initial concentration at time zero, $k$ is the rate constant for degradation of TMQ in day ${ }^{-1} t$ is the time in days, and $C_{t}$ is the concentration of TMQ at incubation time $(t)$. The parameters of the least squares regression line for eqn. 1 are summarized in Table I. The mean ( \pm S.D.) rate constant for drug degradation was $0.0134 \pm 0.0002$ day $^{-1}$ and, therefore, the mean half-life $(0.693 / k)$ for TMQ at $37^{\circ} \mathrm{C}$ in glass was $51.6 \pm 0.8$ days. If one defines the maximum allowable percent drug loss before either drug replacement or pump refill as $10 \%$, then the maximum allowable incubation (or infusion) time between TMQ refill or replacement based on the rate constant of degradation obtained in this study would be 7.9 days.

\section{Products of TMQ degradation}

Since the half-life of TMQ in this preparation at $37^{\circ} \mathrm{C}$ is only 51.6 days, the degradation product(s) would be expected to accumulate in significant amounts even in the relatively short incubation period of 1-2 weeks. Fig. 2 shows a chromatogram (HPLC) of TMQ samples (2.0 $\mu$ linjections) from zero-time, 21-day and 56-day incubations. It is evident from Fig. 2 that as the parent drug peak (B) progressively decreases with increasing incubation time, other earlier eluting peaks (primarily peak A) are increasing. It is to be noted that most of the early eluting peaks (including peak A) are absent or only marginally present in the zero-time or TMQ standard solutions.

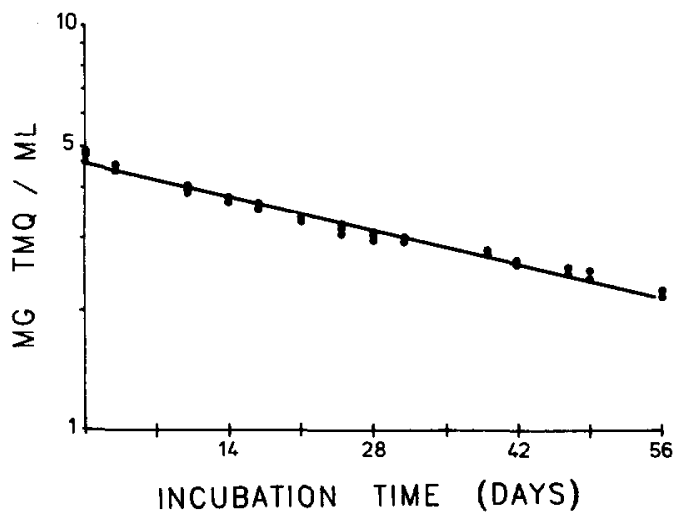

Fig. 3. TMQ concentration versus incubation time (in days). Data have been fitted to a monoexponential equation of the form $C_{t}=C_{0} \exp (-k t)$, with the results: $C_{t}=(4.56 \pm 0.10) \exp -(0.0134 \pm 0.0002) t$ and correlation coefficient $r=0.993 \pm 0.002$. 


\section{TABLE I}

SUMMARY OF PARAMETERS OF THE LEAST SQUARES REGRESSION LINE FOR EQN. 1

Concentration of incubate $=5.00 \mathrm{mg} / \mathrm{ml}$.

\begin{tabular}{lccc}
\hline Drug & \multicolumn{1}{l}{$k$} & $k$ & $\begin{array}{c}t_{1 / 2} \\
\text { (days) }\end{array}$ \\
\hline Trimetrexate & & & \\
Incubate 1 & 4.60 & 0.0136 & 51.0 \\
Incubate 2 & 4.63 & 0.0135 & 51.3 \\
Incubate 3 & 4.45 & 0.0132 & 52.5 \\
Mean \pm S.D. & $4.56 \pm 0.10$ & $0.0134 \pm 0.0002$ & $51.6 \pm 0.8$ \\
\hline
\end{tabular}

Using the diode array UV-VIS detector, UV spectral data were gathered for both TMQ and its major degradation product, peaks B and A in Fig. 2, respectively. These spectral data are shown in Fig. 4. The major degradation product has a distinctly different $U V$ spectrum, with an absorbance maximum at $253 . \mathrm{nm}$ and a secondary peak at $332 \mathrm{~nm}$. TMQ's UV spectrum elicits two primary peaks at 215 and $243 \mathrm{~nm}$ and a small secondary peak at $330 \mathrm{~nm}$.

The GC-MS analysis of the vacuum-dried residues from the zero-time and 56-day incubation samples derivatized with MSTFA revealed a large peak $\left(t_{\mathrm{R}}=5.0\right.$ $\mathrm{min}$ ) in the 56-day sample which was absent in the zero-time sample. Both samples had a large peak eluting at $9.2 \mathrm{~min}$, corresponding to authentic TMQ-bisTMS. When analysed in the EI mode, the mass spectrum of TMQ-bisTMS (Fig. 5) displayed a molecular ion at $m / z 513$ and the typical TMS derivative fragmentation pattern with loss of a methyl group yielding $[\mathrm{M}-15]^{+}$at $m / z 498$, and the prominent [TMS] ${ }^{+}$at $m / z$ 73. The base peak at $m / z 331$ corresponds to cleavage at the anilino-methyl

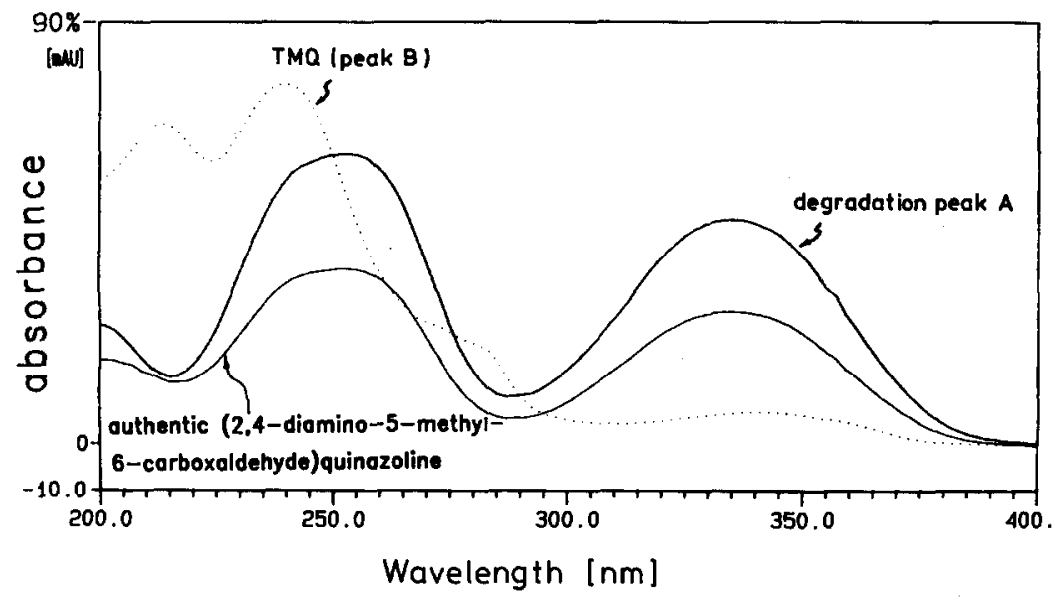

Fig. 4. UV-VIS absorption spectra of TMQ (peak B in Fig. 2), degradation product (peak A in Fig. 2), and authentic AMCQ. 


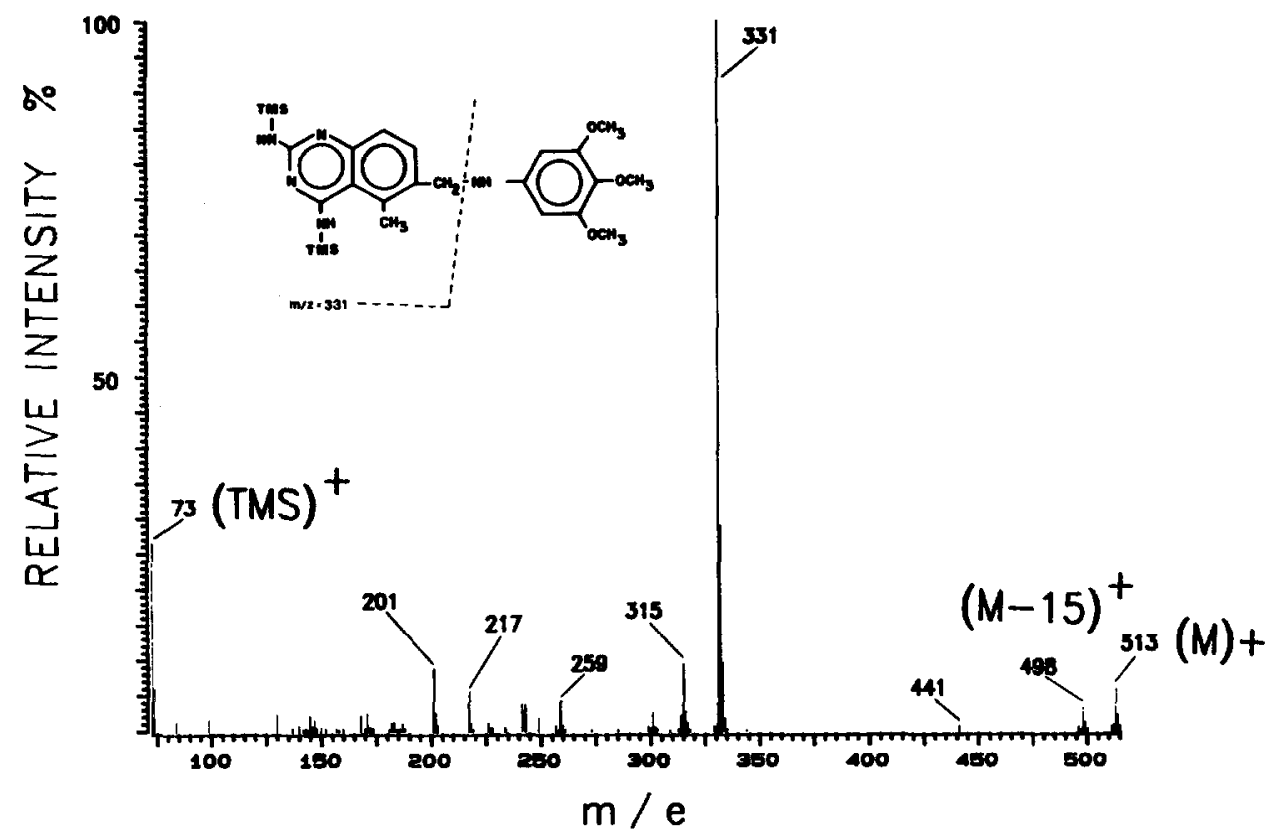

Fig. 5. EI mass spectrum of TMQ-bisTMS (peak B in Fig. 2).
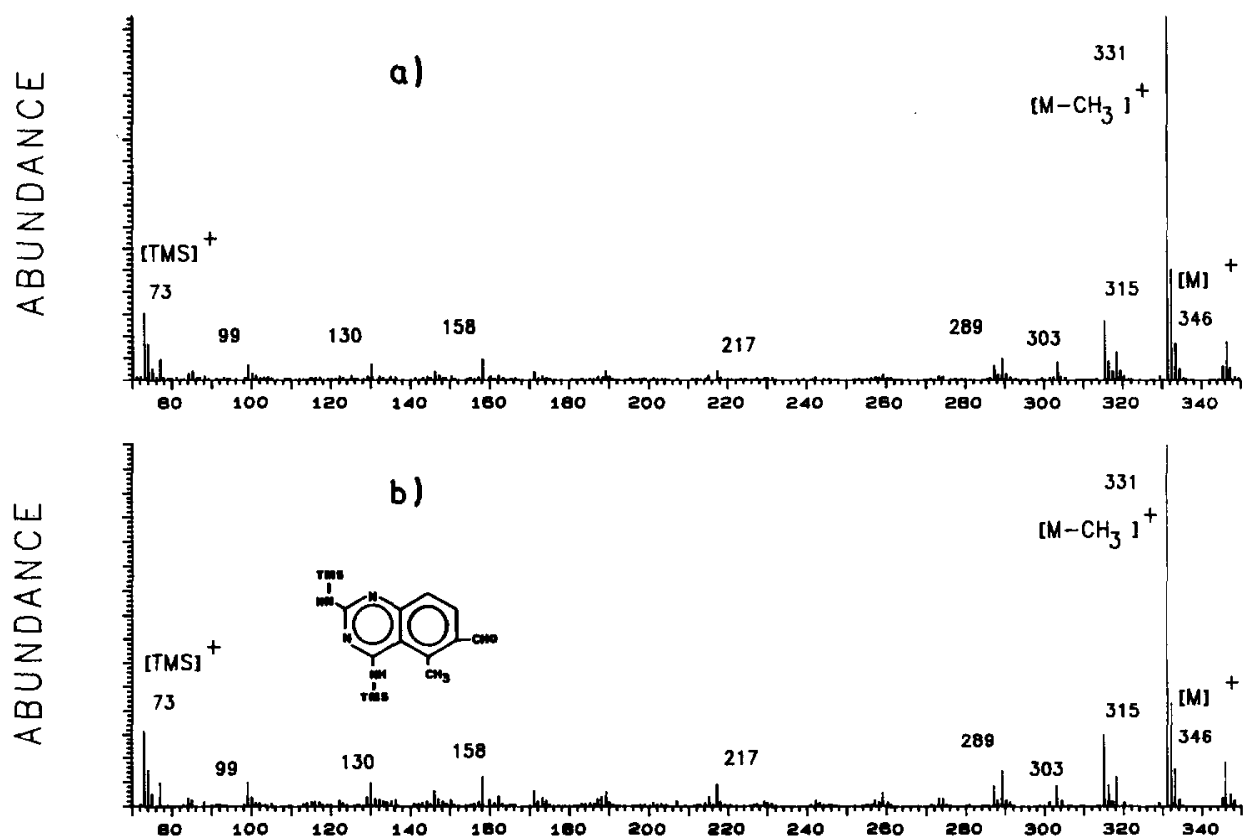

Fig. 6. EI mass spectra of (a) trimethylsilylated degradation product (peak A in Fig. 2), and (b) authentic AMCQ-bisTMS. 
linkage. The degradation product's mass spectrum (Fig. 6a) suggests that its molecular ion is at $m / z 346$, and its base peak is [M-15] ${ }^{+}$at $m / z 331$, in this case due to loss of a methyl group from one of the TMS adducts. Also present were significant peaks at $m / z 345$ and 318 corresponding to the $[\mathrm{M}-1]^{+}$and $[\mathrm{M}-\mathrm{CO}]^{+}$peaks which are characteristic of aromatic aldehydes.

GC-MS analysis of these samples in the methane CI mode resulted in base peaks of TMQ and its degradation product at the corresponding $[\mathrm{M}+1]^{+}$ions $(\mathrm{m} / \mathrm{z}$ 514 and 347, respectively). Also characteristic of methane CI, each mass spectrum included an $[\mathrm{M}+29]^{+}$ion, due to the addition of $\left(\mathrm{C}_{2} \mathrm{H}_{5}\right)$. Finally, the fragmentation patterns were consistent with TMS derivatives showing a significant $[M-15]^{+}$peak. The TMQ-bisTMS CI mass spectrum retained as its second largest peak the ion at $m / z 331$.

Additional evidence for the presence of an aldehyde in the incubated samples was obtained with the 2,4-dinitrophenylhydrazine test for aldehydes and ketones ${ }^{14}$. The zero-time sample tested negative for aldehydes and ketones (no precipitate formation). However, the 56-day sample elicited a strong positive response with the immediate formation of an orange-red precipitate (the insoluble dinitrophenylhydrazone).

The mass spectral and chemical data suggested the identity of the degradation product as AMCQ. Mass spectral (Fig. 6) and UV-VIS absorption spectral (Fig. 4) analysis of authentic AMCQ confirmed this conclusion.

In conclusion, the chemical stability of the pharmaceutical preparation (Trimetrexate glucuronate) of this non classical antifolate has been evaluated. Incubation in sterile, amber glass vials at $37^{\circ} \mathrm{C}$ for 56 days resulted in a degradation rate constant of $0.0134 \pm 0.0002$ day $^{-1}$ and a half-life of $51.6 \pm 0.8$ days. The major degradation product has been identified as AMCQ. Ten percent TMQ degradation would occur by 7.9 days of incubation under these conditions. Therefore, use of this drug preparation in implanted drug delivery systems would necessitate weekly pump refill or replacement if one sets $10 \%$ degradation as the maximum allowed.

\section{ACKNOWLEDGEMENT}

This work was supported in part by NIH Grant No. CA 33825.

\section{REFERENCES}

1 E. F. Elslager and J. Davoll, in R. N. Castle and L. B. Townsend (Editors), Lectures in Heterocyclic Chemistry, Vol. II, Hetero Corp., Orem, UT, 1974, pp. 5-97.

2 J. J. McCormack, in W. Pfleiderer (Editor), Chemistry and Biology of Pteridines, Walter de Gruyter, New York, NY, 1976, p. 125.

3 J. R. Bertino, W. L. Sawicki, B. A. Moroson, A. R. Cashmore and E.F. Elslager, Biochem. Pharmacol., 28 (1979) 1983.

4 H. Diddens, N. Niethammer and R. C. Jackson, Cancer Res., 43 (1983) 5286.

5 J. R. Bertino, Cancer Res., 39 (1979) 293.

6 G. F. Borsa and F. A. Whitmore, Cancer Res., 29 (1969) 737.

7 G. F. Borsa, F. A. Whitmore, F. A. Valeriote, D. Collins and W. R. Bruce, J. Natl. Cancer Inst. (U.S.), 42 (1969) 235.

8 H. E. Skipper, F. M. Schabel, L. B. Mellett, J. A. Montgomery, L. J. Wilkoff, H. H. Lloyd and R. W. Brockman, Cancer Chemother. Rep., 54 (1970) 431. 
9 B. A. Chabner and R. C. Young, J. Clin. Invest., 52 (1973) 1804.

10 H. M. Pinedo, D. S. Zaharko, J. M. Bull and B. A. Chabner, Cancer Res., 37 (1977) 445.

11 D. S. Zaharko, Pharmacological Basis of Cancer Chemotherapie, Williams and Wilkins, Baltimore, MD, 1975, pp. 69-84.

12 F. M. Sirotnak, Pharmacol. Ther. Part A, 8 (1980) 71.

13 W. D. Ensminger and J. W. Gyves, Cancer Treat. Rep., 68 (1984) 101.

14 D. J. Pasto and C. R. Johnson, Organic Structure Determination, Prentice-Hall, Englewood Cliffs, NJ, 1969, p. 382. 